\title{
Erratum to "High-Efficiency Min-Entropy Estimation Based on Neural Network for Random Number Generators"
}

\author{
Na Lv $\mathbb{D}^{1,2,3}$ Tianyu Chen $\mathbb{D}^{1,},{ }^{1,2}$ Shuangyi Zhu, ${ }^{1,2,3}$ Jing Yang, ${ }^{4}$ Yuan Ma, ${ }^{1,2}$ Jiwu Jing, \\ and Jingqiang $\operatorname{Lin}^{1,2}$ \\ ${ }^{1}$ State Key Laboratory of Information Security, Institute of Information Engineering, Chinese Academy of Sciences, \\ Beijing 100093, China \\ ${ }^{2}$ Data Assurance and Communications Security Research Center, Chinese Academy of Sciences, Beijing 100093, China \\ ${ }^{3}$ School of Cyber Security, University of Chinese Academy of Sciences, Beijing 100093, China \\ ${ }^{4}$ China Information Technology Security Evaluation Center, Beijing 100085, China \\ ${ }^{5}$ School of Computer Science and Technology, University of Chinese Academy of Sciences, Beijing 100093, China
}

Correspondence should be addressed to Tianyu Chen; chentianyu@iie.ac.cn

Received 11 October 2020; Accepted 11 October 2020; Published 23 October 2020

Copyright (c) $2020 \mathrm{Na}$ Lv et al. This is an open access article distributed under the Creative Commons Attribution License, which permits unrestricted use, distribution, and reproduction in any medium, provided the original work is properly cited.

In the article titled "High-Efficiency Min-Entropy Estimation Based on Neural Network for Random Number Generators" [1], the author Jing Yang was affiliated to "School of Computer Science and Technology, University of Chinese Academy of Sciences, Beijing 100093, China," and the author Jiwu Jing was affiliated to "China Information Technology Security Evaluation Center, Beijing 100085, China," which is incorrect. The correct affiliations for these authors are as follows.

The author Jing Yang's affiliation is "China Information Technology Security Evaluation Center, Beijing 100085," and the author Jiwu Jing's affiliation is "School of Computer Science and Technology, University of Chinese Academy of Sciences." This mistake was introduced during the production, and Hindawi apologises for causing this error in the article.

The corrected list of affiliations is shown in the author information above.

\section{References}

[1] N. Lv, T. Chen, S. Zhu et al., "High-efficiency min-entropy estimation based on neural network for random number generators," Security and Communication Networks, vol. 2020, Article ID 4241713, 18 pages, 2020. 